\title{
The 6 minute walk in idiopathic pulmonary fibrosis: longitudinal changes and minimum important difference
}

\author{
Jeffrey J Swigris, ${ }^{1}$ Frederick S Wamboldt, ${ }^{2}$ Juergen Behr, ${ }^{3}$ Roland M du Bois, ${ }^{1}$ \\ Talmadge E King, ${ }^{4}$ Ganesh Raghu, ${ }^{5}$ Kevin K Brown ${ }^{1}$
}

${ }^{1}$ Interstitial Lung Disease Program and Autoimmune Lung Center, National Jewish Health, Denver, Colorado, USA ${ }^{2}$ Division of Psychosocial Medicine, National Jewish Health, Denver, Colorado, USA ${ }^{3}$ Department of Internal Medicine I, University of Munich, Germany ${ }^{4}$ Division of Pulmonary and Critical Care Medicine, University of California San Francisco, California, USA ${ }^{5}$ Division of Pulmonary and Critical Care Medicine, University of Washington, Seattle, Washington DC, USA

Correspondence to Dr Kevin K Brown, National Jewish Health, 1400 Jackson Street, Denver, CO 80206, USA;

brownk@njc.org

Received 8 January 2009 Accepted 29 December 2009 Published Online First

8 December 2009

\section{ABSTRACT}

Rationale The response characteristics of the 6 minute walk test (6MWT) in studies of idiopathic pulmonary fibrosis (IPF) are only poorly understood, and the change in walk distance that constitutes the minimum important difference (MID) over time is unknown.

Objectives To examine changes over time in distance walked (ie, 6MWD) during the 6MWT and to estimate the change in distance that constitutes the MID in patients with IPF.

Methods Data from a recently completed trial that included subjects with IPF who completed the 6MWT, Saint George's Respiratory Questionnaire (SGRO) and forced vital capacity (FVC) at 6 and 12 months were used to examine longitudinal changes in 6MWD. Both anchorand distribution-based approaches as well as linear regression analyses were used to determine the MID for 6MWD. The SGRO Total score and FVC were used as clinical anchors.

Main results Among 123 subjects alive and able to complete the 6MWT at both follow-up time points, 6MWD did not change significantly over time $(378.1 \mathrm{~m}$ at baseline vs $376.8 \mathrm{~m}$ at 6 months vs $361.3 \mathrm{~m}$ at 12 months, $p=0.5$ ). The point estimate for the $6 M W V D$ MID was $28 \mathrm{~m}$ with a range of $10.8-58.5 \mathrm{~m}$.

Conclusion In a group of patients with IPF with moderate physiological impairment, for those alive and able to complete a 6MWT, 6MWD does not change over 12 months. At the population level, the MID for 6MWD appears to be $\sim 28 \mathrm{~m}$. Further investigation using other anchors and derivation methods is required to refine estimates of the MID for GMWD in this patient population.

\section{INTRODUCTION}

Idiopathic pulmonary fibrosis (IPF) is a progressive, fatal fibrosing interstitial lung disease that impairs functional status and quality of life. Although recent advances in our understanding of the underlying pathogenesis of IPF have led to the investigation of numerous profibrotic pathways, ${ }^{1}$ no medical treatment has been shown to improve respiratory symptoms or functional status, or to prolong survival.

The 6 minute walk test (6MWT) is a simple and easily performed assessment of a subject's submaximal functional capacity. It measures the distance a patient can walk on a flat surface over a period of $6 \mathrm{~min}$ and is used to measure the response to medical interventions in patients with moderate to severe cardiac or pulmonary disease. ${ }^{2}$ Its reproducibility (with a coefficient of variation of
8\%) appears to be better than the $1 \mathrm{~s}$ forced expiratory volume in patients with chronic obstructive pulmonary disease (COPD),$^{3-6}$ and it has less short-term variability than questionnaire indices of functional status $(22-33 \%)$.

For IPF, the 6 minute walk distance (6MWD) appears highly reproducible (test-retest reliability 0.98 ) over short time intervals (eg, 1-2 weeks), and is highly correlated $(r=0.78)$ with maximal oxygen uptake derived from a maximal cardiopulmonary exercise test. ${ }^{8}$ In two relatively small studies, investigators have examined changes in distance walked during the 6MWT over longer time periods in patients with confidently defined IPF. Tomioka and colleagues ${ }^{9}$ found that $6 \mathrm{MWD}$ declined to a nonsignificant degree $(37.3 \pm 120.9 \mathrm{~m}, \mathrm{p}=0.2)$ from baseline among 32 subjects with IPF who completed a $6 \mathrm{MWT}$ after a median 14 months of follow-up. In a recently completed placebo-controlled study investigating the efficacy of etanercept in the treatment of IPF, the 6MWT was used as a secondary endpoint. In terms of $6 \mathrm{MWD}$, there was no statistically significant difference between the two treatment arms, and subjects in the placebo arm $(\mathrm{n}=41)$ experienced a mean decline of $14.7( \pm 112.5) \mathrm{m}$ $(p=0.5)$ at week 48 , with changes of -2.2 $( \pm 65.80), 2.1( \pm 71.7)$ and $-22.3( \pm 106.8) \mathrm{m}$ at weeks 12,24 and 36 , respectively. ${ }^{10}$

What is not known about the 6MWT in patients with IPF is what distance constitutes the minimum important difference (MID) - the smallest change in distance that patients can perceive as different from the previous test and that would mandate, in the absence of troublesome side effects and excessive costs, a change in management. ${ }^{11}$ Several methods can be used to determine the MID for an outcome measure, but there is much controversy over which ones are best. ${ }^{12} \mathrm{~A}$ common approach is to use multiple methods to derive MID estimates. One - the anchor-based method-calls for a different but related clinical variable to be a so-called anchor; the mean change in the outcome (eg, $6 \mathrm{MWD})$ calculated for subjects who change "minimally" according to that anchor is the MID estimate. There are no restrictions on what variables can serve as anchors; however, any anchor must be clinically relevant and cut-off values for defining minimal change should be sensible. Distributionbased methods, including the effect size, standardised response mean and standard error of measurement, use statistical calculations based solely on study sample data for the outcome variable to derive the MID. 
The goals of this study were to determine the changes in $6 \mathrm{MWD}$ over time in a large, well-defined sample of subjects with IPF with moderate physiological impairment and to use multiple approaches to determine the MID for 6MWD in this population.

\section{METHODS}

\section{Patient population}

Data from the international, prospective, double-blinded, randomised, placebo-controlled, parallel group study investigating the use of bosentan in the treatment of patients with IPF (BUILD-1) were analysed. ${ }^{13}$ Its study design, inclusion and exclusion criteria, and primary results have been previously published. ${ }^{13}$ Briefly, patients were enrolled in BUILD-1 if their IPF diagnosis met American Thoracic Society (ATS)/European Respiratory Society (ERS) consensus guidelines, ${ }^{14}$ if they were diagnosed with IPF between 3 and 36 months prior to enrolment, and if a screening 6MWD was between 150 and $499 \mathrm{~m}$. Patients were excluded if they had a forced vital capacity (FVC) of $<50 \%$ or $>90 \%$ predicted, ${ }^{15}$ a diffusing capacity for carbon monoxide $\left(\mathrm{DL}_{\mathrm{CO}}\right)$ corrected for haemoglobin level $<30 \%$ predicted, ${ }^{16}$ a resting arterial oxygen pressure $\left(\mathrm{PaO}_{2}\right)<55 \mathrm{~mm} \mathrm{Hg}$ (sea level) or $50 \mathrm{~mm} \mathrm{Hg}$ (>1400 m), echocardiographic evidence of severe pulmonary hypertension (systolic pulmonary pressure $>50 \mathrm{~mm}$ $\mathrm{Hg}$ or tricuspid regurgitation velocity $>3.2 \mathrm{~m} / \mathrm{s}$ ) or severe congestive heart failure. The study was approved by the appropriate independent ethics committees or institutional review boards and conducted in accordance with the principles of the Declaration of Helsinki, local laws and guidelines for good clinical practice. All patients provided written, informed consent. Our study sample was comprised of subjects with baseline, 6 and 12 month 6MWD data.

\section{Study protocol}

The 6MWD was measured without supplemental oxygen using a modified 6MWT protocol, conducted in accordance with ATS guidelines, ${ }^{2}$ but terminated prematurely if $\mathrm{SpO}_{2}$ fell below $80 \%$ (pulse oximeter; Nellcor N-595; Pleasanton, California, USA). Technicians conducting the 6MWT were blinded to all questionnaire data.

\section{Assessment tools}

The Saint George's Respiratory Questionnaire (SGRQ) is a selfadministered, obstructive lung disease-specific questionnaire with 50 items comprising three domains (Symptoms, Activity and Impact) and a Total score. Domain and Total scores range from 0 to 100 , with higher scores corresponding to worse healthrelated quality of life.

\section{Statistical analysis}

Descriptive statistics were used for baseline, 6 month and 12 month data. Change over time in $6 \mathrm{MWD}$ was examined by
Table 1 Baseline characteristics of the entire study sample

\begin{tabular}{ll}
\hline & All subjects (N=154) \\
\hline Age & $65.1(8.74)$ \\
M:F & $73: 27$ \\
Height (cm) & $170.0(8.4)$ \\
Weight $(\mathrm{kg})$ & $84.7(15.87)$ \\
FVC (litres) & $2.6(0.71)$ \\
$\mathrm{FVC} \%$ & $67.8(11.84)$ \\
$\mathrm{DL}_{\mathrm{co}}$ & $4.1(1.18)$ \\
$\mathrm{DL}_{\mathrm{co}} \%$ & $41.8(9.48)$ \\
Baseline $6 \mathrm{MWD}$ & $372.9(82.63)$ \\
$150-249 \mathrm{~m}$ & $(\mathrm{n}=15) 200.9(29.3)$ \\
$250-349 \mathrm{~m}$ & $(\mathrm{n}=37) 309.5(28.7)$ \\
$350-450 \mathrm{~m}$ & $(\mathrm{n}=102) 421.7(40.4)$ \\
\hline Data are presented as mean (SD) or percentages for gender. \\
DL $\mathrm{L}_{\text {co, }}$ diffusing capacity for carbon monoxide; $\mathrm{F}$, female; FVC, forced vital \\
capacity; M, male, 6MWD, 6 minute walk distance.
\end{tabular}

using longitudinal data analytic methods. We used SAS PROC MIXED (SAS Institute, Cary, North Carolina, USA) to model mean $6 \mathrm{MWD}$ at each study time point (baseline, 6 and 12 months) while handling the within-subject correlation of repeated measures of $6 \mathrm{MWD}$. Thus, the model yielded least squared means estimates for $6 \mathrm{MWD}$ at each of the three time points, and the null hypothesis of equality among the three mean values for a given variable was tested. Even with mild deviations from normality and particularly for sample sizes $>100$ - the data from this sample of 123 subjects were mildly skewed-PROC MIXED will yield robust estimates.

Next, we stratified subjects according to anchor score changes (SGRQ Total score and FVC) over the two time intervals-from baseline to 6 months as well as $6-12$ months. The SGRO Total was chosen as an anchor because it captures patients' perceptions about their own health status; thus it assesses certain effects of IPF on patient well-being. In a previous study, we determined the MID for the SGRQ Total to be 7 points, with a range of 5-10 points. Thus, for this analysis, we categorised subjects as "unchanged" if the difference in SGRQ Total was within 5 points (exclusive) of the value at the previous time point, as "changed minimally" if the difference in SGRO Total was 5-10 points (exclusive) different from the previous time point, and as "changed more than minimally" if the difference was $\geq 10$ points. For each time interval, the mean change in 6MWD was calculated for subjects stratified according to categorical changes (ie, unchanged, changed minimally or changed more than minimally) in SGRO Total score. To show the ability of SGRO Total to discriminate between subjects whose 6MWD changed over time, we used repeated measures analysis of variance (ANOVA) to compare linear contrasts in mean changes in 6MWD across categories of change in SGRQ Total score. We used this same
Figure 1 Mean 6 minute walk distance (6MWD) in metres at baseline, 6 months and 12 months for the subjects alive and able to complete the 6 minute walk test (6MWT) at all three time points. Dots indicate the mean 6MWD in metres. Whiskers depict $95 \%$ Cls. Mixed model $p=0.5$ for difference between $6 M W D$ at three time points.

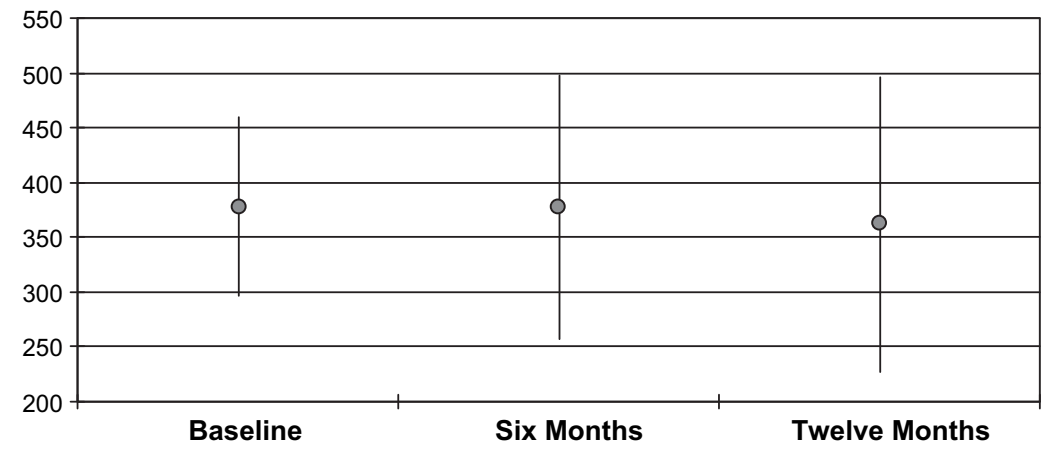


Table 2 Longitudinal change in 6MWD for the subgroup alive and able to complete the 6MWT

\begin{tabular}{rllll}
\hline & Baseline & 6 months & 12 months & p Value \\
\hline Baseline 6MWD & & & & \\
$150-249 \mathrm{~m}(\mathrm{n}=11)$ & $198.3(32)$ & $211.5(127.5)$ & $172.5(118.8)$ & 0.7 \\
$250-349 \mathrm{~m}(\mathrm{n}=28)$ & $311.3(28.5)$ & $279.4(106)$ & $262.9(110.2)$ & 0.13 \\
$350-499 \mathrm{~m}(\mathrm{n}=84)$ & $424.5(39.6)$ & $430.9(77)$ & $419.5(97.5)$ & 0.6
\end{tabular}

Data are presented as mean (SD). $p$ Value from repeated measures ANOVA that compared within-group 6MWD between time points.

$6 \mathrm{MWD}, 6$ minute walk distance; 6MWT, 6 minute walk test.

approach for the FVC anchor: first, stratifying the sample on change in FVC, then calculating mean changes in 6MWD for categories of change in FVC, and finally using repeated measures ANOVA to compare changes in 6MWD across FVC categories. For FVC, we categorised subjects as "unchanged" if the difference in the raw FVC was within 7\% (inclusive) of the value at the previous time point, as "changed minimally" if the difference in the raw FVC value was between $7 \%$ and $12 \%$ (exclusive) of the value at the previous time point, and as "changed more than minimally" if the difference was $\geq 12 \%$. We considered a $7-12 \%$ change in raw FVC as a minimally important change because this range covers $7 \%$ (a so-called marginal change that has been shown to carry prognostic significance in $\operatorname{IPF}^{17}{ }^{18}$ ) as well as $10 \%$ (a commonly used endpoint in trials and in clinical practice ${ }^{14}$ ).

To estimate the MID for $6 \mathrm{MWD}$, we calculated weighted averages for $6 \mathrm{MWD}$ for subjects with minimal change in the SGRQ Total (5-10 points) or FVC anchor (7-12\% from the baseline raw value). We next used the within-patient anchorbased method ${ }^{19}$ to derive another MID estimate for 6MWD. For this method, linear regression is used to examine the relationship between changes in 6MWD (dependent variable) and changes in the anchor-SGRO Total score or FVC\% (independent variables). Lastly, we used distribution-based methods to generate MID estimates. The effect size (referred to as ES and calculated as the change in 6MWD divided by the SD of the baseline 6MWD) is one such method. ${ }^{20}$ For ES, values of 0.2 are considered small effects, 0.5 medium effects, and 0.8 large effects. Although there is no consensus about how or even whether ${ }^{12}$ ES should be used in the estimation of MIDs, some investigators consider 0.5 to correspond to the MID. ${ }^{21}{ }^{22}$ Thus, we derived the change in $6 \mathrm{MWD}$ that would correspond to an ES of 0.5 and used that as an MID estimate. All analyses were performed with SAS version 9.1.3 (SAS Institute), and $p$ values $<0.05$ were considered statistically significant.

\section{RESULTS}

The mean age of the sample was 65 years, and most subjects were men (table 1). Among all subjects able to complete a 6MWT at baseline and at both 6 and 12 month follow-up $(n=123)$, there was no statistical difference in mean $6 \mathrm{MWD}$ between any time point (figure 1). Further, table 2 shows that baseline walk distance appeared not to influence subsequent measures of 6MWD.

Statistically significant relationships were observed between change in 6MWD and change in SGRO Total score or FVC (table 3). Table 4 gives MID estimates from distribution- and anchor-based approaches. The distribution-based approach $(\mathrm{ES}=0.5)$ yielded a higher estimate than the anchor-based approaches. The mean of all MID point estimates yielded a value of $28 \mathrm{~m}$.

\section{DISCUSSION}

For patients with IPF, the 6MWT appears to be a valid reflection of global functional capacity ${ }^{8}$; it is frequently used clinically to assess changes in IPF disease status over time but with few data to support this practice. More importantly, the 6MWT-either the $6 \mathrm{MWT}$ itself, variations thereof or data collected during the $6 \mathrm{MWT}$ (eg, measures of oxygenation)-has been used as an outcome measure in trials enrolling subjects with IPF. $^{23-25}$ However, there are large knowledge gaps regarding certain important aspects of this test in IPF.

In this study, for a select group of well-defined subjects with IPF who were able to walk no less than $150 \mathrm{~m}$ and no more than $499 \mathrm{~m}$ during a baseline 6MWT, and who performed follow-up testing, we found no significant change from baseline in $6 \mathrm{MWD}$ at 6 or 12 months. These data are supported by studies from Tomioka and Raghu who also found no significant change over time frames ranging from about 12 to 14 months. ${ }^{9} 10$

Our study estimates the MID for 6MWD in patients with IPF, which we found to be in the range of $28 \mathrm{~m}$. To our knowledge, only two other groups of investigators have assessed the MID for the 6MWD in patients with IPF. In a study published only in abstract form, Mathai and colleagues divided into two subgroups 20 patients with IPF who were participating in a 1 day support group. Each patient performed a $6 \mathrm{MWT}$, and then each subgroup of 10 patients spent the day together at a support group meeting (details not provided). At the end of the day, each patient was asked to rate his ability to walk relative to the other members in his subgroup. These investigators found that $6 \mathrm{MWD}$ needed to differ by a mean $17.9 \pm 103.6 \mathrm{~m}$ for patients to stop rating themselves as "the same" as other members in their group. Redelmeier and colleagues developed this method, and used it to show that the MID for 6MWD in patients with COPD is $54 \mathrm{~m}$

Table 3 Mean 6MWD for the subgroup alive and able to complete the 6MWT at all three time points stratified by the SGR0 and FVC anchors SGR0 total score

\begin{tabular}{|c|c|c|c|c|c|}
\hline & Worsened $\geq 10$ & Worsened 5-10 & Within 4.9 points & Improved 5-10 & Improved $\geq 10$ \\
\hline Baseline to 6 months & $\begin{array}{l}n=16 \\
-39.0(128.2)\end{array}$ & $\begin{array}{l}n=19 \\
-6.6(77.3)\end{array}$ & $\begin{array}{l}\mathrm{n}=36 \\
8.0(58.6)\end{array}$ & $\begin{array}{l}n=16 \\
15.8(47.1)\end{array}$ & $\begin{array}{l}n=34 \\
2.9(76.7)\end{array}$ \\
\hline $6-12$ months & $\begin{array}{l}n=17 \\
-84.6(111.2)\end{array}$ & $\begin{array}{l}n=20 \\
-20.2(49.0)\end{array}$ & $\begin{array}{l}n=48 \\
-10.4(67.4)\end{array}$ & $\begin{array}{l}n=14 \\
25.9(58.4)\end{array}$ & $\begin{array}{l}\mathrm{n}=24 \\
5.6(57.9)\end{array}$ \\
\hline \multicolumn{6}{|l|}{ FVC } \\
\hline & Declined $\geq 12 \%$ & Declined $7-12 \%$ & No change & Improved 7-12\% & Improved $\geq 12 \%$ \\
\hline Baseline to 6 months & $\begin{array}{l}n=17 \\
-56.1(111.9)\end{array}$ & $\begin{array}{l}\mathrm{n}=24 \\
-20.6(105.4)\end{array}$ & $\begin{array}{l}\mathrm{n}=65 \\
11.7(49.6)\end{array}$ & $\begin{array}{l}n=9 \\
8.8(35.6)\end{array}$ & $\begin{array}{l}n=8 \\
56.8(43.1)\end{array}$ \\
\hline
\end{tabular}

Data are presented as mean (SD). Tests of significance for linear contrast in group effect yielded $p=0.05$ for baseline -6 months and $p=0.0001$ for $6-12$ months for $S G R 0$ Total and $p=0.0003$ for baseline -6 months and $p=0.2$ for $6-12$ months for FVC.

FVC, forced vital capacity; 6MWD, 6 minute walk distance; 6MWT, 6 minute walk time; SGR0, Saint George's Respiratory Questionnaire. 
Table 4 MID estimates for 6MWD

\begin{tabular}{|c|c|c|c|c|c|c|}
\hline Time & $E S=0.5$ & $\Delta \mathrm{FVC}=7-12 \%$ & $\Delta S G R Q t=5-10$ & Regression equation for FVC & Regression equation for SGRQt & Mean \\
\hline Baseline to 6 months & $41.3 \mathrm{~m}$ & $17.4 \mathrm{~m}^{*}$ & $10.8 \mathrm{~m}$ & $\begin{array}{l}\triangle 6 \mathrm{MWD}=2.6+1.9(\Delta \mathrm{FVC}) \\
\text { Corresponding to } 10 \% \text { change in raw } \mathrm{FVC} \text {, } \\
\Delta 6 \mathrm{MWD}=21.6(8.6-33.6)\end{array}$ & $\begin{array}{l}\triangle 6 \mathrm{MWD}=-4+(-1.2)(\Delta S G R Q t) \\
\text { Corresponding to } 7 \text { unit change in SGROt, } \\
\Delta 6 \mathrm{MWD}=12.4(5.4-19.4)\end{array}$ & 20.7 \\
\hline
\end{tabular}

Grand mean $=28$, thus $M I D=28 \mathrm{~m}$.

The $95 \% \mathrm{Cls}$ around the MID estimates from the regression equations are sample size dependent. The point estimates should be viewed as the best estimates of the MID.

*Weighted mean of subjects whose raw FVC changed by $7-12 \%$ over the indicated time interval.

ES, effect size; FVC, forced vital capacity; MID, minimum important difference; 6MWD, 6 minute walk distance; SGROt, Saint George's Respiratory Ouestionnaire SGRO Total.

(95\% CI 37 to $71 \mathrm{~m})$; that is, the mean difference in $6 \mathrm{MWD}$ between subjects who rated themselves as being able to walk either "a little bit better" or "a little bit worse", compared with those who rated themselves as being able to walk "about the same" as other patients, was $54 \mathrm{~m}$. In that study, patients who rated themselves as "a little worse" on average walked $80 \mathrm{~m}$ less than other patients in their group, whereas subjects who rated themselves as "a little better" walked only $30 \mathrm{~m}$ more than other patients in their group-yielding a weighted average of $54 \mathrm{~m}$ among subjects who rated themselves as minimally (or "a little...") different from other patients.

Another method commonly used to determine the MID for an outcome measure involves asking subjects to provide a so-called transition assessment at the time of follow-up testing. For example, at 6 months, subjects (blinded to baseline and followup data) might be asked to report whether they perceived their 6MWD to be "the same as", "a little bit less", "a lot less", "a little bit greater" or "a lot greater" than their baseline walk. The mean 6MWD for subjects reporting a minimal change in perceived 6MWD (ie, "a little bit less" or "a little bit greater") would be the estimate of the MID. Singh and co-investigators used this method to estimate the MID for the incremental shuttle walk test to be $47.5 \mathrm{~m}$ in patients with COPD. ${ }^{26}$ In the only study of the 6MWD MID in IPF published in manuscript format, Holland and her colleagues used global change ratings, receiver operating characteristic (ROC) curves and a distribution-based approach to derive their estimate. ${ }^{27}$ Among 24 subjects with IPF who completed a 6MWT before and after an 8 week exercise programme, the MID was found to be between 29 and $34 \mathrm{~m}$.

No transition assessment was used in the BUILD-1 trial, but we employed a related (and perhaps the most common) method of determining the MID-an anchor-based method. Although any variable can be an anchor, each should meet three criteria: (1) it should be related to the outcome variable; (2) it must possess face validity; and (3) it must be able to be divided into at least three categories: no change, minimal and other. ${ }^{12}$ As one anchor, we selected the SGRQ Total for several reasons: (1) it is a patientoriented or patient-assessed outcome measure and, as such, it asks specifically about patients' perceptions ${ }^{28}$-a notion viewed by many investigators as paramount to deriving the MID for an outcome variable $e^{12}$; (2) IPF impairs-and patients value-the quality of their lives, so the SGRO and other health status questionnaires provide meaningful data in this population ${ }^{29} 30$; and (3) we have shown previously that a 7-point change in SGRO Total score is its MID among patients with IPF. ${ }^{31}$ As the second anchor we chose the FVC because it is perhaps the most widely used physiological measurement to assess IPF severity, and cutoff values for its clinical and prognostic meaningfulness have been established in a number of studies. ${ }^{32-36}$ We elected to use FVC rather than $\mathrm{DL}_{\mathrm{CO}}$ because of the greater intrinsic variability in $\mathrm{DL}_{\mathrm{CO}}$ measures as well as our inability to define confidently the clinically significant range for its minimum change.
There are a number of limitations to this study. As is often the case, the distribution-based method yielded higher estimates for the MID than the anchor-based methods. ${ }^{37}$ The selected population included only patients with IPF who could walk $>150 \mathrm{~m}$ but $<499$ m during a 6 MWT at baseline-and the overwhelming majority could walk at least $350 \mathrm{~m}$. Furthermore, data from subjects who died, or who were unable to complete the 6MWT for other reasons, were not included in our analyses; this could introduce bias. Thus, inferences drawn here may not be applicable to extremely debilitated patients with IPF in the latter stages of the disease or to more fit patients with IPF in the earlier stages of disease. However, the results of this study may have important implications for both IPF investigators who plan to power future IPF studies for change in 6MWD as well as clinicians who prognosticate future changes in functional status. While the pattern of decline in 6MWD over time is unclear, if a linear decline were assumed, one might expect a $30 \mathrm{~m}$ decline in 6MWD to occur, on average, at 31 months of follow-up (data not shown). It has been recommended that multiple anchors (in multiple studies) be used to generate a range for the MID for any outcome variable. ${ }^{12}$ Although this is but one study, we derived the MID for 6MWD by using two clinically meaningful anchors as well as by employing distribution-based methods. Furthermore, our estimate is nearly identical to the estimate from the only other published study to examine the 6MWD MID in IPF. Finally, it must be recognised that the MID estimate here is to be used at the population level; that is, the mean change in 6MWD that is considered clinically important in a population is "often much less" than the change in 6MWD that would allow a practitioner to be confident that a change within an individual patient is outside of the inter-test variability. ${ }^{37}$

In conclusion, data from this study provide the first systematic examination of 12 month longitudinal changes in $6 \mathrm{MWD}$ as a prospectively acquired, primary outcome variable in a welldefined subset of patients with IPF, as well as an estimate of the MID for 6MWD in patients with this disease. It appears as though the MID for 6MWD is smaller for IPF than for COPD, but future confirmatory studies should be performed to estimate the MID for 6MWD in IPF.

Acknowledgements The authors would like to acknowledge all of the investigators involved in the BUILD-1 trial: Ishaar Ben-Dov, Charles Chan, Jean-Francois Cordier, James Dauber, Joao De Andrade, Adaani Frost, Thomas Geiser, Marilyn Glassberg, Jeffrey Golden, Gary Hunninghake, Sanjay Kalra, Lisa Lancaster, Robert Levy, Keith Meyer, Joachim Mueller-Quernheim, Paul Noble, Christophe Pison, Charles Poirier, Milton Rossman, Paola Rottoli, Gerd Staehler, Athol Wells, Gordon Yung and David Zisman. The authors also wish to acknowledge the study coordinators and nurses for their hard work and all of the study subjects for their generous contributions.

Funding Actelion Pharmaceuticals funded the performance of the underlying BUILD-1 trial that investigated the efficacy of bosentan in the treatment of idiopathic pulmonary fibrosis. No additional external funding was obtained. All authors had full access to all the data in this study. The corresponding author had final responsibility for the decision to submit the paper for publication. 
Competing interests JB, RMduB, TEK, GR and KKB served on the steering committee for the BUILD-1 trial, sponsored by Actelion Pharmaceuticals. JJS has served as a paid consultant to Actelion Pharmaceuticals. FSW has no competing interests to declare.

Ethics approval This study was approved by the appropriate independent ethics committees or institutional review boards.

Provenance and peer review Not commissioned; externally peer reviewed.

\section{REFERENCES}

1. Selman M, King TE, Pardo A. Idiopathic pulmonary fibrosis: prevailing and evolving hypotheses about its pathogenesis and implications for therapy. Ann Intern Med 2001;134:136-51.

2. ATS Committee on Proficiency Standards for Clinical Pulmonary Function Laboratories. ATS statement: guidelines for the six-minute walk test. Am J Respir Crit Care Med 2002:166:111-17.

3. Butland RJ, Pang J, Gross ER, et al. Two-, six-, and 12-minute walking tests in respiratory disease. Br Med J 1982;284:1607-8

4. Knox AJ, Morrison JF, Muers MF. Reproducibility of walking test results in chronic obstructive airways disease. Thorax 1988;43:388-92.

5. Guyatt GH, Pugsley SO, Sullivan MJ, et al. Effect of encouragement on walking test performance. Thorax 1984;39:818-22.

6. Leger LA, Lambert J. A maximal multistage 20-m shuttle run test to predict V02 max. Eur J Appl Physiol Occup Physiol 1982:49:1-12.

7. Guyatt G, Sullivan $M$, Thompson $P$, et al. The 6-minute walk: a new measure of exercise capacity in patients with chronic heart failure. CMAJ 1985; 132:919-23.

8. Eaton T, Young P, Milne D, et al. Six-minute walk, maximal exercise tests: reproducibility in fibrotic interstitial pneumonia. Am J Respir Crit Care Med 2005;171:1150-7.

9. Tomioka H, Imanaka K, Hashimoto K, et al. Health-related quality of life in patients with idiopathic pulmonary fibrosis-cross-sectional and longitudinal study. Intern Med 2007; 46:1533-42

10. Raghu G, Brown KK, Costabel U, et al. Treatment of idiopathic pulmonary fibrosis with etanercept: an exploratory, placebo-controlled trial. Am J Respir Crit Care Med 2008:178:948-55

11. Juniper $\mathbf{E}$, Guyatt G, Willan $A$, et al. Determining a minimal important change in a disease-specific quality of life questionnaire. J Clin Epidemiol 1994;47:81-7.

12. Hays RD, Farivar SS, Liu H. Approaches and recommendations for estimating minimally important differences for health-related quality of life measures. Copd 2005:2:63-7.

13. King TE Jr, Behr J, Brown KK, et al. BUILD-1: a randomized placebo-controlled trial of bosentan in idiopathic pulmonary fibrosis. Am J Respir Crit Care Med 2008;177:75-81.

14. American Thoracic Society/European Respiratory Society. International multidisciplinary consensus classification of the idiopathic interstitial pneumonias Am J Respir Crit Care Med 2000;161:646-64.

15. Morris J, Koski A, Johnson L. Spirometric standards for the healthy non-smoking adults. Am Rev Respir Dis 1971;103:57-67.

16. Crapo R, Morris A. Standardized single breath normal values for carbon monoxide diffusing capacity. Am Rev Respir Dis 1981;123:185-9.

17. Zappala C, Latsi $P$, Nicholson AC, et al. Marginal declines in FVC levels are associated with increased mortality in idiopathic pulmonary fibrosis. Thorax 2007:175:A143.
18. Du Bois RM, Albera C, Costabel U, et al. Categorical declines in percent predicted forced vital capacity are associated with a graded risk of death in patients with idiopathic pulmonary fibrosis. Chest 2008;134:S20003. http://meeting.chestpubs.org/ cgi/content/abstract/134/4/s20003

19. Puhan MA, Frey M, Buchi S, et al. The minimal important difference of the hospita anxiety and depression scale in patients with chronic obstructive pulmonary disease. Health Qual Life Outcomes 2008;6:46.

20. Cohen J. Statistical analysis for the behavioral sciences 2nd edn. New Jersey: Lawrence Erlbaum Associates Inc, 1988.

21. Walters SJ, Brazier JE. What is the relationship between the minimally important difference and health state utility values? The case of the SF-6D. Health Qual Life Outcomes 2003:1:4.

22. Norman GR, Sloan JA, Wyrwich KW. Interpretation of changes in health-related quality of life: the remarkable universality of half a standard deviation. Med Care 2003;41:582-92.

23. Azuma A, Nukiwa T, Tsuboi E, et al. Double-blind, placebo-controlled trial of pirfenidone in patients with idiopathic pulmonary fibrosis. Am J Respir Crit Care Med 2005;171:1040-7.

24. Nishiyama 0, Kondoh $Y$, Kimura T, et al. Effects of pulmonary rehabilitation in patients with idiopathic pulmonary fibrosis. Respirology 2008;13:394-9.

25. Nishiyama 0, Taniguchi $\mathrm{H}$, Kondoh Y, et al. Dyspnoea at 6-min walk test in idiopathic pulmonary fibrosis: comparison with COPD. Respir Med 2007;101:833-8.

26. Clark M, Cooper B, Singh S, et al. A survey of nocturnal hypoxaemia and health related quality of life in patients with cryptogenic fibrosing alveolitis. Thorax 2001;56:482-86

27. Holland $\mathbf{A E}$, Hill CJ, Conron $\mathrm{M}$, et al. Small changes in six-minute walk distance are important in diffuse parenchymal lung disease. Respir Med 2009:103:1430-35.

28. Abrams D. Analysis of a life-satisfaction index. J Gerontol 1976;24:470.

29. Swigris JJ, Kuschner WG, Jacobs SS, et al. Health-related quality of life in patients with idiopathic pulmonary fibrosis: a systematic review. Thorax 2005; 60:588-94

30. Swigris JJ, Stewart AL, Gould MK, et al. Patients' perspectives on how idiopathic pulmonary fibrosis affects the quality of their lives. Health Qual Life Outcomes 2005;3:61

31. Swigris JJ, Brown KK, Behr J, et al. The SF-36 and SGRO: validity and first look at minimum important differences in IPF. Respir Med 2009 [Epub ahead of print]

32. Collard HR, King TE Jr, Bartelson BB, et al. Changes in clinical and physiologic variables predict survival in idiopathic pulmonary fibrosis. Am J Respir Crit Care Med 2003; 168:538-42.

33. Flaherty KR, Andrei AC, Murray S, et al. Idiopathic pulmonary fibrosis: prognostic value of changes in physiology and six-minute-walk test. Am J Respir Crit Care Med 2006;174:803-9.

34. Flaherty KR, Mumford JA, Murray S, et al. Prognostic implications of physiologic and radiographic changes in idiopathic interstitial pneumonia. Am J Respir Crit Care Med 2003;168:543-8

35. King TE Jr, Tooze JA, Schwarz MI, et al. Predicting survival in idiopathic pulmonary fibrosis: scoring system and survival model. Am J Respir Crit Care Med 2001; 164:1171-81.

36. Latsi PI, du Bois RM, Nicholson AG, et al. Fibrotic idiopathic interstitial pneumonia: the prognostic value of longitudinal functional trends. Am J Respir Crit Care Med 2003;168:531-7

37. Wise RA, Brown CD. Minimal clinically important differences in the six-minute walk test and the incremental shuttle walking test. COPD 2005;2:125-9. 